\title{
Nursing Roles And Functions In The Inpatient Neurorehabilitation Of Stroke Patients: A Literature Review
}

Fariba Tabari ${ }^{1}$

${ }^{1}$ Assistant Professor, School of Nursing and Midwifery, Tehran University of M edical Sciences, Tehran, Iran.

Stroke is the third largest cause of death and largest cause of adult disability in the United Kingdom and United States. The World Health Organization (WHO)[1] estimates 15 million people worldwide will have a stroke annually; this represents a major health burden. The purpose was to explore the nursing roles and functions in the Inpatient Neurorehabilitation of Stroke Patients.

\section{Method:}

In this review study, an online search among articles published from 2000 to 2016 was conducted through CINAHL, PubMed, Science Direct, Elsevier, SID, Iran Medex, Magiran and Google scholar databases using key words; "Inpatient Neurorehabilitation of Stroke Patients", "Nursing Roles and Functions". The protocol of York University Guide was used to select the articles.

\section{Results:}

In total, 15 articles were used from 40 articles that were initially obtained from the search, from which, 6 were Iranian articles and the rest were non-Iranian articles. The articles indicated that, nursing roles and functions in the Inpatient Neurorehabilitation of Stroke Patients can be divided into the factors related to patient role, interdisciplinary cooperation, feedback to the staff about the patient's progress, to provide emotional Support for patients and relatives.

\section{Conclusions:}

The contribution that nurses with stroke rehabilitation skills can make to effective stroke care was understood. However, The nurse considers the individual's needs working collaboratively with the patient and their families to involve them in a meaningful way with decision making and their recovery.

Key words: Nursing Roles, Nursing Functions, Inpatient Neurorehabilitation of Stroke Patients, A Literature Review

DOI: $10.7575 /$ aiac.abcmed.ca1.65

Published Date: February 2017

Peer-review is under responsibility of the 9th Iranian Stroke Congress.

Published by Australian International Academic Centre, Australia

This published work is open access under the CC BY license.

Available online at www.abcmed.aiac.org.au 